\title{
The Thermo-Dependence Effects on Buoyancy Convection Heat Transfer in a Square Enclosure, Filled with Shear-Thickening Power-Law Fluids and Submitted to Cross Uniform Heat
}

\author{
M. Kaddiri ${ }^{1}{ }^{\text {* }}$, M. Naimi ${ }^{1}$, A. Raji ${ }^{1}$, M. Hasnaoui ${ }^{2}$ \\ ${ }^{1}$ Faculty of Sciences and Technologies, Laboratory of flows and Transfers Modeling (LAMET), Sultan Moulay Slimane University, \\ Beni-Mellal, Morocco \\ ${ }^{2}$ Faculty of Sciences Semlalia, Laboratory of Fluid Mechanics and Energetics (LMFE), Cadi Ayyad University, Marrakech, Morocco
}

\section{Email address:}

mouradkadiri@usms.ma (M. Kaddiri),naimi@fstbm.ac.ma (M. Naimi)

\section{To cite this article:}

M. Kaddiri, M. Naimi, A. Raji, M. Hasnaoui. The Thermo-Dependence Effects on Buoyancy Convection Heat Transfer in a Square Enclosure, Filled with Shear-Thickening Power-Law Fluids and Submitted to Cross Uniform Heat. International Journal of Fluid Mechanics \& Thermal Sciences. Vol. 1, No. 2, 2015, pp. 30-35. doi: 10.11648/j.ijfmts.20150102.13

\begin{abstract}
Two-dimensional steady-state buoyancy driven flows of thermo-dependent shear-thinning power-law fluid confined in a square cavity, submitted to cross uniform heat fluxes, has been conducted numerically using a finite difference technique. The parameters governing the problem are the thermo-dependence number $\mathrm{m}(0 \leq m \leq 10)$ and the ratio between the heat flux imposed on the vertical walls and that imposed on the horizontal ones represented by a $(0 \leq a \leq 1)$, while the flow behavior index $\mathrm{n}$ is fixed at $(n=1.4)$ and the Rayleigh number at $\left(R_{a}=5000\right)$. The effects of these parameters on the flow structure and heat transfer characteristics have been analyzed.
\end{abstract}

Keywords: Natural Convection, Heat Transfer, Thermo-Dependent Behavior, Non-Newtonian Fluid

\section{Introduction}

Thermal buoyancy convection is a flow resulting from density variations within a non-isothermal fluid under the gravity effect. Such a phenomenon is of importance in various domains, which attracted many worldwide researchers, through the decades, to investigate it in many geometrical configurations and under various boundary conditions. Useful literature review can be found in the article and book by Ostrach [1] and Gebhart et al. [2], respectively, where most of the fluids considered are of Newtonian behavior.

However, most of materials that are of interest in a variety of manufacturing processes, exhibit non-Newtonian behaviors, which implies that, the shear stress is not proportional to the shear rate [2]. According to Skelland [3], non-Newtonian fluids can be classified into three main groups, which are namely purely viscous, viscoelastic and time-dependent fluids. Purely viscous non-Newtonian fluids can be divided into two categories: shear-thinning or pseudo-plastic fluids and shear-thickening or dilatant fluids. For the formers (shear-thinning fluids), the viscosity is a decreasing function of the rate of shear. This property is specific to some complex solutions like ketchup, whipped cream, blood, paint, and nail polish. It is also a common property of polymer solutions and molten polymers. Pseudo-plasticity can be proved for example by shaking a bottle of ketchup, which leads to an unpredictable change in the viscosity of the content. The viscous force causes it to go from being thick like honey to flowing like water. For the latter (shear-thickening fluids), the viscosity increases with the rate of shear. The dilatant effect can readily be seen with a mixture of cornstarch and water, which acts in a counterintuitive way when thrown against a surface.

On the other hand, during their thermal processing, non-Newtonian products are often subjected to natural convection flow, that is why such a phenomenon involving such fluids has been broadly investigated during the last three decades, but owing to their complex rheological behavior and their particular non-isothermal flow conditions further investigations should be undertaken in this area, especially when we know that most of them display strong temperature dependence, which constitutes another challenging problem to deal with. In fact, this effect makes difficult analytical 
investigations even when flowing within simple geometries. Usually, the dependence of the properties on temperature is the most important effect. This leads to nonlinearity in the governing equations and couples the flow with the energy transport. Thus, the solution of the governing equations and the interpretation of experimental results become more involved than for constant property circumstances. Average constant property values at different reference conditions are frequently employed to simplify the solution. However, most manufacturing processes require the solution of the full variable-property problem for accurate predictions of the resulting transport [2].

To our best knowledge, most of the reported studies on natural convection involving non-Newtonian fluids ignored the dependence of the effective viscosity on temperature (thermo-dependence in other words). This can be a serious assumption, since in many cases this dependence has a significant influence on flow and heat transfer as proved, earlier, experimentally by Scirocco et al. [4] and numerically by Shin and Cho [5] whose results, of local Nusselt numbers for a polyacrylamide (Separan AP-273) solution, show $70-300 \%$ heat transfer enhancement over those of a constant-property fluid. Indeed, according to these authors, the effective viscosity thermo-dependence engenders non-negligible modifications of the velocity fields and heat transfer. This is expressed by an augmentation of the velocity magnitude near the heated wall, where the viscosity is seriously reduced because of the high temperatures in this region, and the heat exchange rate.

Most of studies having dealt with such an effect, for non-Newtonian fluids, were concerned with mixed convection in ducts. For instance, in the case of shear-thinning fluids, Sirocco et al. [4] and Shin and Cho [5] observed experimentally and numerically, respectively, that the enhancement of the heat transfer from the heated wall is due to an increase of the velocity gradient near the wall, which they attributed to the combined effect of the thermo-dependence and the shear-thinning behavior of the effective viscosity. Such an effect was also observed by Shin et al. [5] and Sohn et al. [6] for a viscoelastic fluid and, recently, by Nouar [7] for a viscoplastic fluid. For natural convection phenomenon in such media, the literature review does not show an important number of investigations carried out in this area, especially for simple geometries such as square and rectangular cavities. Among the few studies conducted in this context, we can cite that of Turki [8], who found that, for power-law fluids filling a rectangular cavity differentially heated from the vertical sides, the consistency thermo-dependence affects substantially the flow structure and the local heat transfer but not significantly the overall one. Lately, Solomatov and Barr $[9,10]$ examined numerically such an effect, for the same types of fluids as those considered by Turki [8], on the onset of the Rayleigh-Bénard convection and found that a decrease of the viscosity with the temperature anticipates the convection onset.

It appears from what precedes that more work on the issue is called for. Therefore, our goal is to contribute to a better understanding of the thermo-dependence effects on thermal buoyancy convection in such media. Accordingly, a numerical study is performed to investigate the temperature-dependent viscosity effect on natural convection flow and heat transfer in a square cavity confining non-Newtonian power-law fluids and subjected to cross uniform densities of heat flux.

\section{Mathematical Formulation}

\subsection{Problem Statement and Viscosity Model}

The geometry under consideration is sketched in Fig. 1. It consists of a two-dimensional square enclosure of size $H^{\prime} \times H^{\prime}$ subjected to cross uniform densities of heat flux, q' and $\mathrm{aq}^{\prime}$.

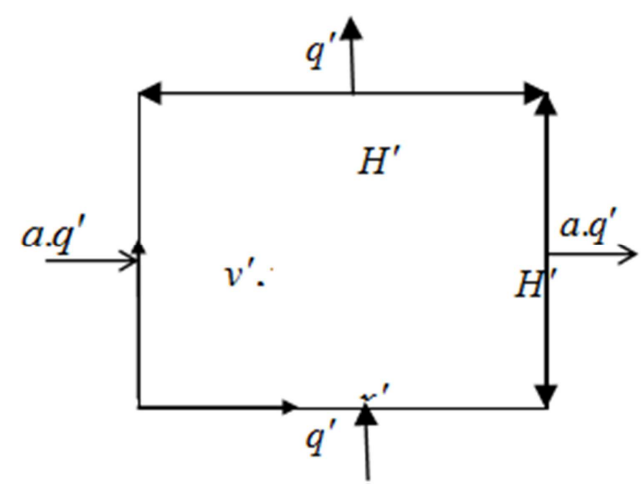

Figure 1. Sketch of the geometry and coordinates system.

The non-Newtonian fluids considered here are those whose rheological behaviors can be approached by the power-law model, due to Ostwald-de Waele, which, in terms of laminar effective viscosity, can be written as follows:

$$
\mu_{a=k_{T}}^{\prime}\left[2\left(\left(\frac{\partial u^{\prime}}{\partial x^{\prime}}\right)^{2}+\left(\frac{\partial v^{\prime}}{\partial y^{\prime}}\right)^{2}\right)+\left(\frac{\partial u^{\prime}}{\partial y^{\prime}}+\frac{\partial v^{\prime}}{\partial x^{\prime}}\right)^{2}\right]^{\frac{\left(n_{T}-1\right)}{2}}
$$

The two empirical parameters $\mathrm{n}_{T}$ and $\mathrm{k}_{T}$ appearing in (1), are the flow behavior and consistency indices, respectively. They are, in general, functions of the temperature, but in most of cases the temperature-dependence of $\mathrm{n}_{T}$ can be ignored $\left(\mathrm{n}_{T}=n\right)$ since it is weak compared to that of $\mathrm{k}_{T}[4,7]$, which is described by the Frank-Kamenetski exponential law [11]:

$$
k_{T}=k e^{-b\left(T^{\prime}-T_{r}{ }^{\prime}\right)}
$$

reflecting the viscosity diminution with the temperature, where $\mathrm{b}$ is an exponent related to the flow energy activation and the universal gas constant, and $T^{\prime}{ }_{r}$ is a reference temperature.

Note that for $\mathrm{n}=1$ the behavior is Newtonian and the consistency is just the viscosity. For $0<n<1$, the effective viscosity decreases with the amount of deformation and the behavior is shear-thinning. Conversely, for $n>1$, the viscosity increases with the amount of shearing, which implies that, the fluid behavior is shear-thickening. 


\subsection{Governing Equations and Boundary Conditions}

On the basis of the assumptions commonly adopted in natural convection problems and using the characteristic scales $H^{\prime}, H^{\prime 2} / \alpha, \alpha / H^{\prime}, \alpha / H^{\prime 2}, q^{\prime} H^{\prime} / \lambda$ and $\alpha$, which correspond respectively to length, time, velocity, vorticity, temperature and stream function, the dimensionless governing equations for Boussinesq-temperature-dependent viscosity fluids, written in terms of vorticity, $\Omega$, temperature, $\mathrm{T}$, and stream function, $\psi$, are as follows:

where

$$
\begin{gathered}
\frac{\partial \Omega}{\partial t}+\frac{\partial(u \Omega)}{\partial x}+\frac{\partial(v \Omega)}{\partial y}=P_{r}\left[\mu_{a} \nabla^{2} \Omega+2 \vec{\nabla} \mu_{a} \cdot \vec{\nabla} \Omega\right]+S_{\Omega}, \\
\frac{\partial T}{\partial t}+\frac{\partial(u T)}{\partial x}+\frac{\partial(v T)}{\partial y}=\nabla^{2} T
\end{gathered}
$$

and

$$
\nabla^{2} \psi=-\Omega
$$

where

$$
\begin{aligned}
& u=\frac{\partial \psi}{\partial \mathrm{x}}, v=-\frac{\partial \psi}{\partial \mathrm{y}}, \Omega=\frac{\partial v}{\partial \mathrm{x}}-\frac{\partial u}{\partial \mathrm{y}}, \\
& S_{\Omega}=P_{r}\left[\left[\frac{\partial^{2} \mu_{a}}{\partial x^{2}}-\frac{\partial^{2} \mu_{a}}{\partial y^{2}}\right]\left[\frac{\partial v}{\partial \mathrm{x}}+\frac{\partial \mathrm{u}}{\partial \mathrm{y}}\right]-2 \frac{\partial^{2} \mu_{a}}{\partial \mathrm{x} \partial \mathrm{y}}\left[\frac{\partial \mathrm{u}}{\partial \mathrm{x}}-\frac{\partial \mathrm{v}}{\partial \mathrm{y}}\right]\right]+P_{r} R_{a} \frac{\partial \mathrm{T}}{\partial \mathrm{x}} \\
& \text { and } \mu_{a=}=\mathrm{e}^{-\mathrm{mT}}\left[2\left(\left(\frac{\partial u}{\partial x}\right)^{2}+\left(\frac{\partial v}{\partial y}\right)^{2}\right)+\left(\frac{\partial u}{\partial y}+\frac{\partial v}{\partial x}\right)^{2}\right]^{\frac{(n-1)}{2}}
\end{aligned}
$$

For the present problem, the appropriate non-dimensional boundary conditions are:

$$
\begin{gathered}
u=v=\psi=\frac{\partial T}{\partial x}+a=0 \text { for } x=0 \text { and } x=1 \\
u=v=\psi=\frac{\partial T}{\partial y}+1=0 \text { for } y=0 \text { and } y=1
\end{gathered}
$$

Note that the major disadvantage of this formulation lies in the fact that $\Omega$ is unknown at the boundaries. To overcome such a difficulty, the Woods formulation has been adopted for stability and accuracy reasons [12].

In addition to the flow behavior index, $n$, and the ratio between heat fluxes, $a$, three other dimensionless parameters appear in the above equations, namely, the Pearson, generalized Prandtl and Rayleigh numbers defined, respectively, as:

$$
\begin{gathered}
m=-\frac{1}{k_{T}} \frac{d k_{T}}{d T}=-\frac{d \operatorname{Ln}\left(k_{T} / k\right)}{d T}, \\
P_{r}=\frac{(k / \rho){H^{\prime}}^{2-2 n}}{\alpha^{2-n}} \text { and } R_{a}=\frac{g \beta H^{\prime 2 n+2} q^{\prime}}{(k / \rho) \alpha^{n} \lambda}
\end{gathered}
$$

The Pearson number (8), which is a new dimensionless quantity taking place in this study, measures the effect of temperature change on the effective viscosity.

\subsection{Heat Transfer}

The steady solution has been used to calculate the average Nusselt number in the horizontal and vertical directions, respectively, defined as:

$$
\begin{aligned}
& N u_{h}=\frac{a q^{\prime} H^{\prime}}{\lambda \overline{\Delta T_{v}{ }^{\prime}}}=\frac{\mathrm{a}}{\overline{\Delta T_{v}}} \\
& N u_{v}=\frac{q^{\prime} H^{\prime}}{\lambda \overline{\Delta T_{h}{ }^{\prime}}}=\frac{1}{\overline{\Delta T_{h}}}
\end{aligned}
$$

where $\overline{\Delta T_{v}}$ is the average temperature difference between the two vertical walls and $\overline{\Delta T_{h}}$ is the average temperature difference between the two horizontal walls.

Here, given the nature of the cross- thermal, the global Nusselt number is introduced by the following equation:

$$
N u_{g}=N u_{h}+N u_{v}
$$

to explain the heat exchange between the thermally active walls.

\subsection{Heatlines Formulation}

The visualization of the paths followed by the heat flow through the enclosure requires the use of the heatlines concept, which consists of lines of constant heat function, $\mathrm{H}$, that are defined, according to Kimura and Bejan [13], from the following equations

$$
\frac{\partial H}{\partial y}=u T-\frac{\partial T}{\partial x},-\frac{\partial H}{\partial x}=v T-\frac{\partial T}{\partial y}
$$

whose derivation, with respect to $\mathrm{x}$ and $\mathrm{y}$, and combination give rise to

$$
\frac{\partial^{2} H}{\partial x^{2}}+\frac{\partial^{2} H}{\partial y^{2}}=-\frac{\partial v T}{\partial x}+\frac{\partial u T}{\partial y}
$$

To obtain the boundary conditions associated with (13), an integration of (12), along the four cavity walls, is necessary, which gives:

$$
\begin{gathered}
H(0, y)=H(0,0) \text { for } x=0 \\
H(x, 1)=H(0,1)-x \text { for } y=1 \\
H(1, y)=H(1,1) \text { for } x=1 \\
H(x, 0)=H(1,0)+1-x \text { for } y=0
\end{gathered}
$$

Finally, the solution of (13) yields the values of $H$, in the computational domain, whose contour plots provide the heatline patterns. Note that only the differences between the values of $\mathrm{H}$ are required instead of its intrinsic ones, which offers the possibility to choose $H(0,0)=0$ as an arbitrary reference value for $\mathrm{H}$.

\section{Solution Procedure}

The two-dimensional governing equations have been discretized using the second order central finite difference methodology with a regular mesh size. The integration of (3) and (4) has been performed with the Alternating Direction Implicit method (ADI), originally used for Newtonian fluids and successfully experimented for non-Newtonian power-law fluids [8,14-15].To satisfy the mass conservation, (5) has been 
solved by a Point Successive Over Relaxation method (PSOR) with an optimum relaxation factor calculated by the Frankel formula [12]. A grid of $81 \times 81$ has been required for obtaining adequate results. At each time step, $\delta \mathrm{t}$, which has been chosen between $10^{-7}$ and $10^{-4}$ (depending on the values of the parameters a and $\mathrm{m}$ ), the convergence criterion

$\left[\frac{\sum_{i, j}\left|\psi_{i, j}^{k+1}-\psi_{i, j}^{k}\right|}{\sum_{i, j}\left|\psi_{i, j}^{k+1}\right|}\right]<10^{-4}$ has been satisfied for $\psi$, where $\psi_{i, j}^{k}$ is the value of the stream function at the node $(i, j)$ for the kth iteration level.

The numerical results from the code have been validated using the benchmark data of de Vahl Davis [16], Turki [8] and Ouertatani [17] for natural convection of Newtonian and non-Newtonian fluids in square enclosures with differentially heated vertical walls and an excellent agreement was obtained (see Table 2 of Ref. [18] and Ref. [19] ).

\section{Results and Discussion}

As was reported in the past by [15], the convection is rather insensitive to $\mathrm{Pr}$ variations, provided that this parameter is large enough as it is the case for the non-Newtonian fluids and for a large category of fluids having a Newtonian behavior. Therefore, Pr is not considered as an influencing parameter in this study and the simulations are conducted with $P_{r} \rightarrow \infty$, i.e. by neglecting the inertia terms on the left hand side of (3) owing to their negligible contribution. To examine the cross fluxes and the thermo-dependency effects, the results presented here are related to $\mathrm{a}=0,0.2,0.5,0.7,0.9$ and 1 , $m=0$ and $10, n=1.4$ and $R_{a}=5.10^{3}$.

Hence, as can be seen from Fig. 2, displaying streamlines (left), isotherms (middle) and heatlines (right), the flow is, in general, unicellular and clockwise, but loses its symmetry with an increasing $\mathrm{m}$ for all the values of a. Also, the streamlines become more crowded in the region neighboring the left lower corner, which means that the flow is intensified as a result of the viscosity decrease in such a region, giving rise to a stagnation zone which tends to be reduced near the right upper corner and to be extended next to the left upper one with an increasing a, while for $m=0$, the effect of a is such that the streamlines become almost parallel to the central part of each wall.

As for the isotherms, they seem to be closely spaced and less distorted in the stagnation region when $\mathrm{m}$ passes from 0 to 10 depending on a, whose increase leads to their rotation in the clockwise direction.

On the other hand, in order to have a microscopic description of the heat transfer process, which is different from the conventional Nusselt number that describes macroscopically such a phenomenon, a heatlines analysis is required. Hence, with comparison to the iso-consistent case $(m=0)$, the heatlines corresponding to the case $(m=10)$ present more distortion, which indicates that the path followed by the heat flow to reach the cold wall is more complicated in the rheological sub-layer. Therefore, the heat transfer is expected to be deteriorated in such a situation. Like the isotherms, an increase of a leads to a deviation of the heatlines in the clockwise direction whatever the value of $\mathrm{m}$.
Moreover, Fig. 3, in which are depicted the variations of $N u_{g}$, show that this quantity increase with a. In addition, the same figure shows an increase in heat transfer with $\mathrm{m}$, and this is clearer if a increases.

In addition, fig. 4-5 presenting the profiles of the stream function, $\psi$, (left) and temperature, T, (right), at mi-height of the cavity along the horizontal direction, $\mathrm{x}$ and along the vertical direction, $y$ respectively, complete the understanding of the thermo-dependency effects on the dynamic and thermal fields. In fact, an increase of $m$ causes displacement of hollow $\psi$ to the left, and a flattening trend, approaching the cold wall, thus justifying the presence of a stagnant zone. These results are also approved by the linearity of the curve $\mathrm{T}$ close to the cold wall.
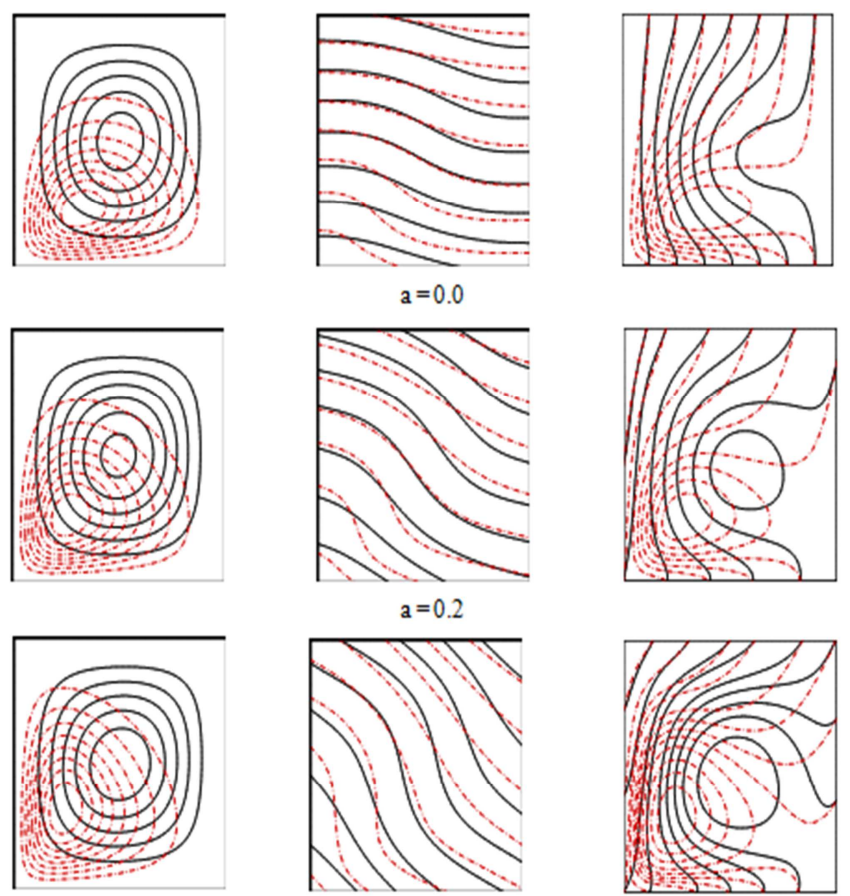

$\mathrm{a}=0.2$
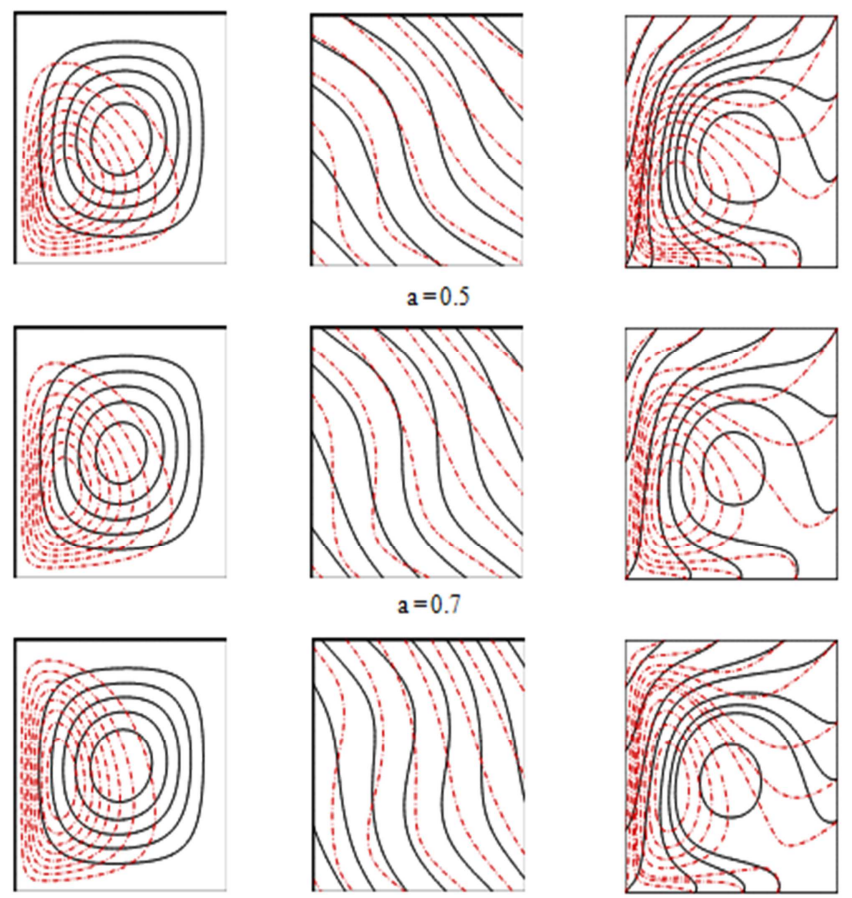

$a=1.0$

Figure 2. Streamlines (left), isotherms (medium) and heatlines (right) for $R_{a}=5.10^{3}, n=1.4$ and $m=0$ (black solid line), $m=10$ (red dashdot line) and various value of $a$. 


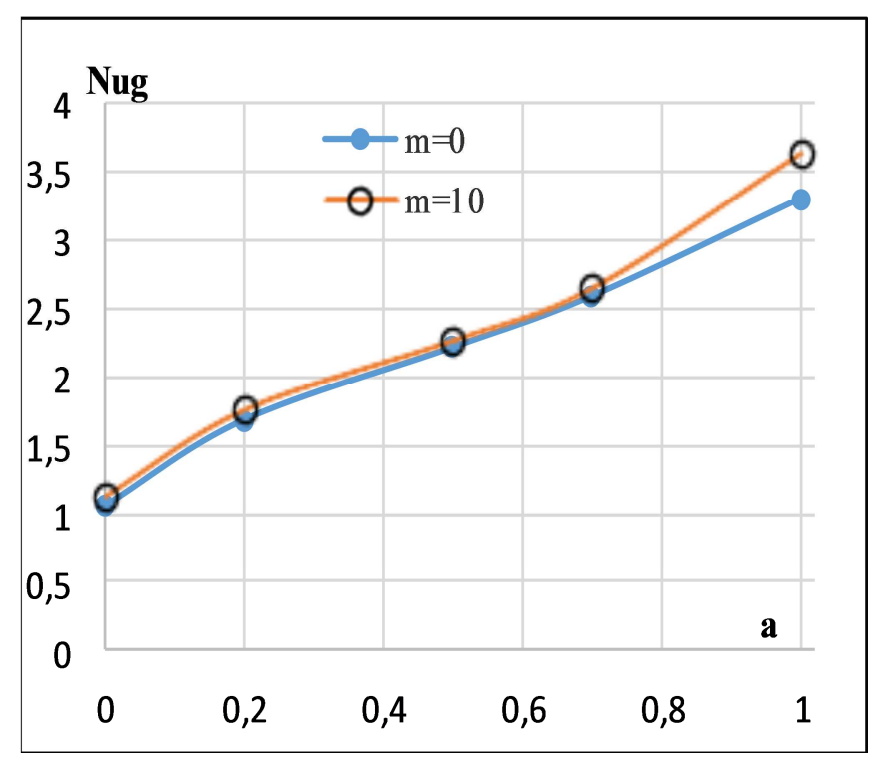

Figure 3. Evolution of the global Nusselt number according to a for $R_{a}=$ 5. $10^{3}, n=1.4, m=0$, and $m=10$.
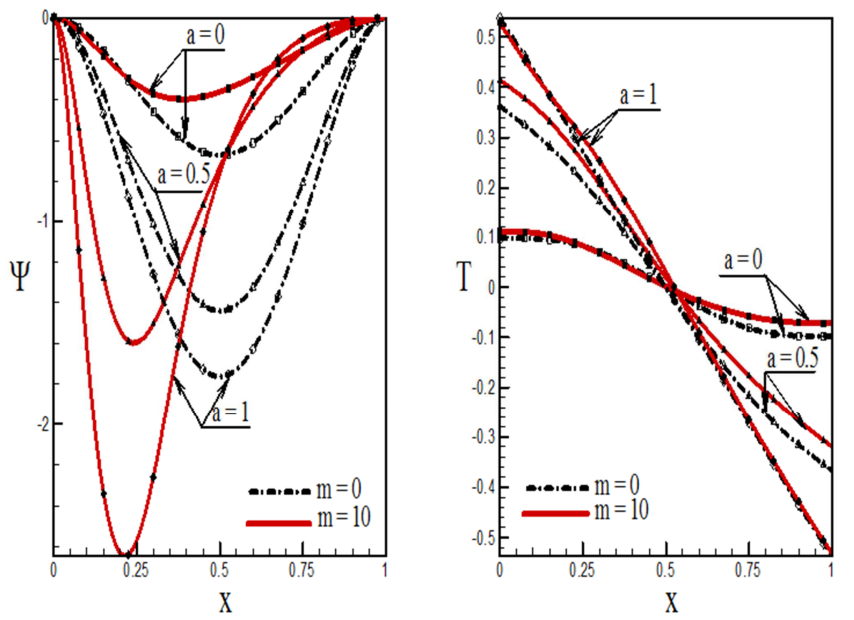

Figure 4. profiles of the stream function, $\psi$, (left) and temperature, T, (right), at mi-height of the cavity along the horizontal direction, $x$.
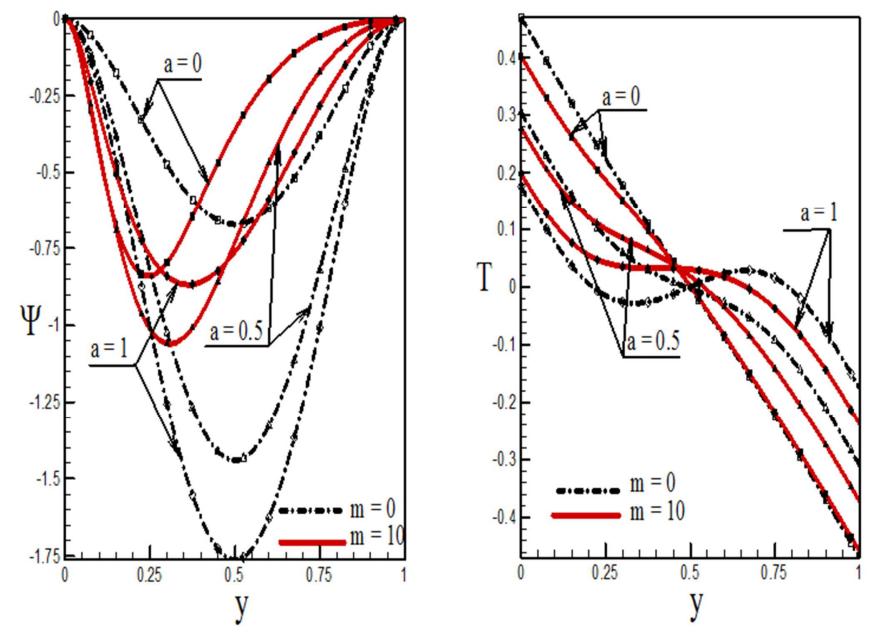

Figure 5. profiles of the stream function, $\psi$, (left) and temperature, T, (right), at mi-height of the cavity along the horizontal direction, $y$.

\section{Conclusion}

A numerical investigation of steady thermal convection in a square enclosure, filled with shear- thickening power-law fluids and submitted to cross uniform heat fluxes, is performed. The exponential model, due to Frank-Kamenetski, for the viscosity variation with the temperature, is used. The study is focused particularly on combined effects of the ratio between the cross heat fluxes and the thermo-dependency parameter (Pearson number) on the flow and thermal fields, and the resulting heat transfer. It emerges that the thermo-dependent behavior affects natural convection heat transfer depending on the proportion of the cross heat fluxes.

\section{Nomenclature}

b: temperature coefficient. g: acceleration due to gravity $\left(\mathrm{m} / \mathrm{s}^{2}\right)$

$H^{\prime}$ : height or width of the enclosure (m)

$\mathrm{k}$ : consistency index for a power-law fluid at the reference temperature $\left(\mathrm{Pa} \cdot \mathrm{s}^{n}\right)$

$\mathrm{m}$ : thermo-dependence number

$\mathrm{n}$ : flow behavior index for a power-law fluid at the reference temperature

Nuh: the horizontal average Nusselt number.

Nuv: the vertical average Nusselt number.

Nug: the global average Nusselt number.

Pr: generalised Prandtl number.

$q^{\prime}$ : constant density of heat flux $\left(\mathrm{W} / \mathrm{m}^{2}\right)$

Ra: generalised Rayleigh number.

$\mathrm{T}$ : dimensionless temperature, $\left(=\left(T^{\prime}-T_{r}^{\prime}\right) / \Delta T^{*}\right)$

$T_{r}^{\prime}$ : reference temperature at the geometric centre of the enclosure $(\mathrm{K})$

$\Delta T^{*}$ : characteristic temperature $\left(=q^{\prime} H^{\prime} / \lambda\right) \quad(\mathrm{K}) \quad(u, v)$

dimensionless horizontal and vertical velocities $\left(=\left(u^{\prime}, v^{\prime}\right) /\left(\alpha / H^{\prime}\right)\right)$

$(x, y)$ : dimensionless horizontal and vertical coordinates $\left(=\left(x^{\prime}, y^{\prime}\right) / H^{\prime}\right)$

Greek symbols

$\alpha$ : thermal diffusivity of fluid at the reference temperature $\left(m^{2} / s\right)$

$\beta$ : thermal expansion coefficient of fluid at the reference temperature $(1 / K)$

$\lambda$ : thermal conductivity of fluid at the reference temperature $\left(\mathrm{W} / \mathrm{m} \cdot{ }^{\circ} \mathrm{C}\right)$

$\mu$ : dynamic viscosity for a Newtonian fluid at the reference temperature $(\mathrm{Pa} \cdot \mathrm{s})$

$\mu_{a}$ : dimensionless effective viscosity of fluid.

$\rho$ : density of fluid at the reference temperature $\left(\mathrm{kg} / \mathrm{m}^{3}\right)$ 
$\Omega$ : dimensionless vorticity, $\left(=\Omega^{\prime} /\left(\alpha / H^{\prime 2}\right)\right)$

$\psi:$ dimensionless stream function, $\left(=\psi^{\prime} / \alpha\right)$

Superscript

': dimensional variables

Subscripts

a: effective variable

max: maximum value

$\mathrm{r}$ : reference value taken at the cavity centre

\section{References}

[1] S. Ostrach, "Natural convection in enclosures," J. Heat Transfer. 1988. 110. P. 1175-1190.

[2] B. Gebhart, Y. Jaluria, R. L. Mahajan, and B. Sammakia, "Buoyancy-induced flows and transport," chap. 16, Hemisphere, Washington, DC, 1988.

[3] A.H.P. Skelland, Non-Newtonian flow and heat transfer, Wiley, New York; 1967.

[4] V. Scirocco, R. Devienne, and M. Lebouché, "Ecoulement laminaire et transfert de chaleur pour un fluide pseudo-plastique dans la zone d'entrée d'un tube, ” Int. J. Heat Mass Transfer, 28(1), pp. 91-99, 1985.

[5] S. Shin, Y.I. Cho, "Laminar heat transfer in a rectangular duct with a non-Newtonian fluid with temperature dependent viscosity," International Journal of Heat and Mass Transfer, vol. 37, no. 1, pp. 19-30, 1994

[6] C.H. Sohn, S.T. Ahn and S. Shin, "Heat transfer behavior of a temperature dependent viscoelastic nonNewtonian fluid with buoyancy effect in 2:1 rectangular duct, " Int. comm. Heat Mass Transfer, 2000, 27(2), pp. 159-168.

[7] C. Nouar, "Thermal convection for a thermodependent yield stress fluid in an axisymmetric horizontal duct, " Int. J. Heat Mass Transfer, 2005, 48, pp. 5520-5535.

[8] S. Turki, Contribution to Numerical Study of Natural and Mixed Convection Heat Transfers in Confined Non-Newtonian Fluids, Ph.D. Thesis, CNAM, Paris, France, 1990.

[9] V.S. Solomatov, and A.C. Barr, "Onset of convection in fluids with strongly temperature-dependent power-law viscosity, " Physics of the Earth and Planetary Interiors, 155, pp.140-145, 2006.
[10] V.S. Solomatov and A.C. Barr, "Onset of convection in fluids with strongly temperature dependent power-law viscosity: 2 . Dependence on the initial perturbation," Physics of the Earth and Planetary Interiors, 2007, 165, pp. 1-13.

[11] N. J. Balmforth and A. Provenzale, Geophysical Aspects of Non-Newtonian Fluid Mechanics, vol. 582 of Liberal National Party, Springer, 2001.

[12] P.J. Roache, Computational Fluid Dynamics, New Mexico: Hermosa Publishers, 1982.

[13] S. Kimura and A. Bejan, "The Heatline Visualization of Convective Heat Transfer," ASME Journal of Heat Transfer, volume 105, pages 916-919, 1983.

[14] H. Ozoe, and S.W. Churchill, "Hydrodynamic stability and natural convection in Ostwald-De Waele and Ellis fluids: the development of a numerical solution, " AIChE J., 18, pp. 1196-1207, 1972.

[15] M. Lamsaadi, M. Naïmi, and M. Hasnaoui, "Natural convection of non-Newtonian power-law fluids in a shallow horizontal rectangular enclosure uniformly heated from the side, " Energy Conversion and Management, 47, pp. 2535-2551, 2006.

[16] De Vahl Davis, G. "Natural convection of air in a square cavity: a bench mark numerical solution," Int. J. Num. Method Fluids, 1983, 3, pp. 249-264.

[17] N. Ouertatani, N. Ben Cheikh, B. Ben Beya, and T. Lili, "Numerical simulation of two-dimensional Rayleigh-Benard convection in an enclosure," Comptes Rendus, vol. 336, no. 5,pp. 464-470, 2008.

[18] M. Kaddiri, M. Naïmi, A. Raji and M. Hasnaoui, "Thermal convection within a square cavity filled with non-Newtonian power-law fluids and differentially heated with uniform heat fluxes, " International Scientific Journal for Alternative Energy and Ecology, Vol. 88, $\mathrm{N}^{\circ}$ 8, pp. 57-69, 2010.

[19] M. Kaddiri, M. Naïmi, A. Raji and M. Hasnaoui, "Rayleigh-Bénard convection of non-Newtonian power-law fluids with temperature-dependent viscosity," ISRN Thermodynamics, Vol. 2012, Article ID 614712, 10 pages, 2012, doi.10.5402/2012/614712. 